Théologiques

Théologiques

\title{
Sagesse, folie et bonheur en Qohélet 10,1
}

\section{Jean-Jacques Lavoie}

Volume 16, numéro 2, 2008

L'individualisation de la relation religieuse

URI : https://id.erudit.org/iderudit/001721ar

DOI : https://doi.org/10.7202/001721ar

Aller au sommaire du numéro

\section{Éditeur(s)}

Faculté de théologie et de sciences des religions, Université de Montréal

\section{ISSN}

1188-7109 (imprimé)

1492-1413 (numérique)

Découvrir la revue

\section{Citer cet article}

Lavoie, J.-J. (2008). Sagesse, folie et bonheur en Qohélet 10,1. Théologiques, 16(2), 177-196. https://doi.org/10.7202/001721ar

\section{Résumé de l'article}

Qo 10,1 est un texte qui ne nécessite aucune correction textuelle. Du point de vue de l'analyse structurelle, Qo 9,18b-10,1a est un commentaire critique de Qo 9,18a, tandis que Qo 10,1b s'oppose à Qo 9,17. L'idée dominante de ce verset peut se résumer comme suit: Qo 10,1 relativise la valeur traditionnelle de la sagesse. Plus précisément, Qo 9,18b-10,1a évoque le monde politico-militaire qui a le pouvoir de détruire le bonheur, tandis que Qo 10,1b souligne qu'un peu

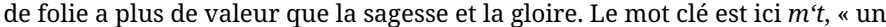
peu ". Il rappelle, non sans ironie, que ni la sagesse ni la folie ne peuvent garantir le bonheur et la sécurité (cf. Qo 7,16-17). 


\title{
Sagesse, folie et bonheur en Qohélet 10,1
}

\author{
Jean-Jacques LAVOIE* \\ Sciences des religions \\ Université du Québec à Montréal
}

Qo 10,1 est un verset qui pose d'énormes problèmes, aussi bien du point de vue de la critique textuelle que de la traduction - et donc de l'interprétation. Le présent texte vise à porter un nouveau regard critique sur ce verset énigmatique. Dans ce dessein, nous nous demanderons d'abord à quels versets ce passage est relié. La réponse à cette question n'est pas sans importance, car elle conditionne le sens que l'on donne à ce passage. Puis, tout en examinant les principales variantes textuelles, nous ferons une analyse littéraire de chacun des mots de ce verset. L'ensemble de cette démarche nous permettra de mieux cerner l'originalité du message que Qo 10,1 nous livre sur la valeur relative de la sagesse et de la folie, et sur la précarité du bonheur.

\section{Critique structurelle}

La délimitation de la péricope ne fait pas l'unanimité parmi les exégètes. D'aucuns rattachent 10,1 à ce qui précède, identifiant une péricope, soit en 9,13-10,1 (Krüger 2004, 178 ; Forti 2005, 242), soit en 9,13-15; 9,1610, 1 (Loader 1979, 58; 1986, 113), soit en 9,17-10,1 (Fox 1999, 298 ; Schwienhorst-Schönberger 2004, 481; Shields 2006, 208). Mais quelques exégètes font de 10,1 le début d'une nouvelle péricope qui se rattache davantage aux v. 2-3 qu'à ce qui précède (Whybray 1989, 150; Vilchez

\footnotetext{
Jean-Jacques Lavoie est professeur titulaire au Département des sciences des religions de l'Université du Québec à Montréal. De 2000 à 2006, il a été directeur de Frontières, revue d'études interdisciplinaires sur la mort et le deuil. Outre de nombreux articles scientifiques sur la tradition sapientiale, mentionnons parmi ses récentes publications: Proverbes. Nouvelle traduction (en collaboration avec Pierre Alferi, Gallimard, 2004), annotation des livres de Job, Siracide, Tobit, Judith et Esther (hébreu et grec) dans La Bible expliquée (Société biblique française, 2004), et collaboration au Nouveau vocabulaire biblique, Paris/Montréal, Bayard/Médiaspaul, 2004.
} 
Lindez 1994, 372-373; Rose 1999, 413). Lauha considère également 10,1-3 comme une unité littéraire. Toutefois, il estime que 9,18a est une glose et il déplace 9,18b entre 10,1a et 10,1b $(1978,175,179,181)$. De son côté, Brandscheidt est d'avis que 10,1-19 constitue une grande unité littéraire qui traite de deux thèmes: le pouvoir destructeur du mal $(10,1-11)$ et la folie (10,12-19); toutefois, puisque les v. 2 à 4 du chapitre 10 sont considérés comme des compléments tardifs et que 9,17-18 et 10,1 sont attribués au second rédacteur, elle reconnaît qu'un lien unit ces trois versets (1999, 130 et 210). Sans postuler une genèse hypothétique et inutilement complexe, quelques exégètes jugent également que 10,1 est relié à la fois à ce qui précède et à ce qui suit. Les délimitations proposées par ces derniers sont toutefois nombreuses : 9,13-10,3 (Zimmerli 1980, 225), 9,13-10,4 (Seow 1997, 321), 9,17-10,3 (Strobel 1967, 148), 9,17-10,4 (Ogden 1980, 32-33 ; D’Alario 1993, 160) ou encore 9,17-10,7 (Lamparter 1955, 122-123).

Du point de vue de la macro-structure, 9,13-10,4 et 10,5-20 constituent deux grandes unités qui sont chacune introduites par le verbe $r$ 'yty. Par ailleurs, la répétition de la racine $n w \underline{h}(9,17 \mathrm{a}$ et $10,4 \mathrm{~b})$ et du verbe $m s ̌ l$ $(9,17 \mathrm{~b}$ et $10,4 \mathrm{a})$ marque clairement une inclusion. La reprise de la racine $\underline{b} t$ ' en $9,18 \mathrm{~b}$ et $10,4 \mathrm{~b}$ confirme la délimitation de cette micro-structure. En ce qui concerne 10,2, il introduit une nouvelle opposition entre deux types de personne: le $\underline{h} k m$ et le $k s y l$. Ce v. 2 est rattaché au v. 3 par la triple reprise du mot $l b$. Par ailleurs, 9,17-18 poursuit indéniablement la réflexion du v. 16 qui conclut par un bref commentaire le récit fictif des v. 13-15. Le v. 17, en effet, reprend le verbe $\check{s}^{\prime}{ }^{\prime}$ du v. 16. Ainsi, 10,1 fait partie d'une petite unité qui est structurée autour d'une double opposition: la valorisation de la sagesse $(9,16 a .17-18 a)$ et la critique d'une telle valorisation (9,16b.18b-10,1). Si l'on inclut le v. 16 qui conclut le récit fictif de 9,13-15, le texte se présente comme suit:

A 9,16a: comparaison en «mieux vaut» (avec twb mn)

B 9,16b : critique débutant avec un $w$, «mais »

C 9,17: comparaison en «plus que» (avec $m)$

A' 9,18a: comparaison en «mieux vaut» (avec twb mn) 
B’ 9,18b-10,1a : critique débutant avec un $w$, «mais »

C' 10,1b: comparaison en "plus que " (avec $m)$.

Par ailleurs, si l'on n'inclut pas le v. 16 qui sert de conclusion au récit qui commence en 9,13, le texte est toujours structuré autour de la même opposition, mais sous la forme d'un chiasme:

A 9,17: comparaison en "plus que » $(\operatorname{avec} m)$

B 9,18a: comparaison en «mieux vaut» (avec twb mn)

B' 9,18b-10,1a : critique introduite par un $w$, «mais »

A' 10,1b: comparaison en "plus que » (avec $m)$.

En résumé, 9,17-10,1 est un texte structuré en chiasme autour d'une double opposition: d'une part, entre une comparaison qui affirme la supériorité de la sagesse (A) et une autre qui affirme la supériorité de la folie (A'), et, d'autre part, entre un dit de préférence qui valorise la sagesse (B) et un commentaire critique introduit par «mais ", qui illustre sa valeur relative en soulignant la précarité du bonheur (B'). Bien entendu, pour justifier cette interprétation, il nous faut sans plus tarder analyser chacun des mots de 10,1 .

\section{Critique littéraire}

Qohélet 10,1a

Le texte massorétique du v. 1a se lit comme suit:

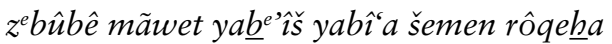

Une traduction littérale des deux premiers mots donnerait «des mouches de mort». Cette expression ambiguë a été comprise de maintes façons. Quelques exégètes estiment qu'il faut rattacher le yod qui se trouve à la fin du mot $z b w b$ au début du mot mwt. Selon eux, ymwt est une proposition relative asyndétique qui doit se comprendre soit comme un verbe qui fait référence à une mouche qui meurt (Glasser 1970, 148), soit comme un substantif qui fait référence à une seule mouche morte (Lauha 1978, 179; Seow 1997, 311-312; Fox 1999, 301; Shields 2006, 209). Bien qu'elles soient ingénieuses, ces interprétations sont invraisemblables, car aucune variante dans la tradition textuelle n'atteste cette lecture. 
D'aucuns transforment le substantif $m w t$ en adjectif et traduisent l'expression zbwby mwt par le pluriel "mouches mortes" (Traduction oecuménique de la Bible 1975, 1630; Bible de Jérusalem 1998, 1109) ou par le singulier "mouche morte" (Dahood 1952, 42; Sacchi 2005, 204) ou encore "vermine putréfiée" (Brandscheidt 1999, 194). Bien que le mot «vermine» ne soit pas le bon équivalent de $z b w b$, d'un strict point de vue grammatical, cette transformation du substantif «mort» en adjectif n'est pas impossible (voir, par exemple, les expressions $b g d y ~ h q d \check{s}$, "les vêtements saints », en Ex 29,29, m'zny tsdq, "balance juste », en Lv 19,36, $m l k y \underline{b} s d$, «rois cléments », en $1 \mathrm{R} 20,31$; etc.). Quant à ceux qui lisent le singulier $z b w b$, ils justifient leur correction en notant que le verbe qui suit est au singulier. Dahood en déduit que le yod final est un ajout dû à une confusion orthographique. Bien qu'elle soit mineure, cette correction est tout aussi injustifiée que la précédente, car tous les manuscrits hébreux et les anciennes versions lisent unanimement le pluriel zbwby: muiai $\left(\mathrm{LXX}^{1}\right)$, $d b b$ ', avec les deux points suscrits qui indiquent le pluriel (Peshitta $\left.{ }^{2}\right)$ et muscee (Vulgate ${ }^{3}$ ). Quant aux verbes qui suivent et qui sont au singulier, on le verra, ils ne nous obligent aucunement à modifier le texte massorétique.

Parmi ceux qui maintiennent le texte hébreu, certains traduisent $z b w b y$ mwt par «mouches mourantes " (Gordis 1968, 314; Rose 1999, 416) ou «mouches moribondes» (Vilchez Lindez 1994, 372). Pour justifier cette traduction, il est possible de la comparer aux expressions 'nšy-mwt et 'yš mwt qui désignent un ou des hommes voués à la mort (2 S 19,29 et $1 \mathrm{R}$ 2,26). Telle était déjà la traduction de la Vulgate: muscae morientes, «mouches mourantes». Par contre, dans son commentaire de l'Ecclésiaste, Jérôme avait traduit plus littéralement par musca mortis, "mouches de mort » (Jérôme 1959, 332).

Seuls deux exégètes ont proposé de rendre l'expression zbwby mwt par "mouche venimeuse », soit au singulier (Delsman 1991, 35), soit au pluriel (Kahn 1989, 1050), supposant ainsi qu'il s'agit d'une espèce de mouches dangereuses, une espèce qui provoque la mort. Par ailleurs, il ne s'agit pas vraiment d'une nouveauté, car la LXX a déjà traduit $m w t$ par le participe actif pluriel du verbe thanatoõ, "faire mourir » : muiai thanatousai, «des

1. Nous travaillons avec l'édition critique établie par Rahlfs (1935).

2. Nous travaillons avec l'édition critique établie par Lane (1979). Plusieurs manuscrits syriaques ( $8 \mathrm{aI}, 9 \mathrm{cI}, 10 \mathrm{cI}, 11 \mathrm{cI}$ ) ajoutent le comparatif ${ }^{\prime} k$, "comme ", devant le mot «mouches": " comme des mouches".

3. Nous travaillons avec l'édition critique de la Biblia Sacra iuxta latinam Vulgatam versionem (1957). 
mouches faisant mourir ${ }^{4}$ ». Telle est aussi la compréhension du Targum qui paraphrase comme suit: $k d y b b$ ' dgrym mwt' b'lm', "c'est comme une mouche qui cause la mort dans le monde» (Taradach et Ferrer 1998, 55). À notre avis, la traduction par «mouches meurtrières » est bien justifiée d'un point de vue grammatical, car la Bible hébraïque contient de nombreux autres exemples où le mot $m w t$ est employé pour désigner ce qui est meurtrier: $k l y$-mwt, les «armes de mort ", c'est-à-dire les "armes meurtrières » (Ps 7,14); mšbry-mwt, les «briseurs de mort », image qui renvoie à la puissance des vagues qui entraînent la mort (2 S 21,5 et Ps 18,5 corrigé); $m w q \check{s} y$ mwt, les "pièges de mort ", c'est-à-dire les «pièges meurtriers " (2 S 22,6; Ps 18,6; Pr 13,14; 14,27), etc.

Saracino a même suggéré d'y voir une espèce spécifique qui porte le nom du dieu ougaritique de la mort: «Mouches de Môt » (Saracino 1982, 339-340). Il est vrai que le mot $m w t$ peut bel et bien aussi faire allusion à la mort personnifiée (voir, par exemple, 2 S 22,5-6; Ps 18,5-6; 116,3; $\operatorname{Pr} 13,14 ; 14,27)$, mais force est de constater que l'expression zbwby mwt en 10,1 est démythisée. Par ailleurs, les exégètes n'ont pas remarqué qu'en dehors de $2 \mathrm{R}$ 2,2.3.6.16, où le mot $z b w b$ apparaît dans l'expression Baal Zeboub, littéralement "Baal de la mouche ${ }^{5}$ ", le mot $z b w b$ n'apparaît qu'en Is 7,18, à côté de l'abeille. Or, les deux insectes évoquent une double attaque militaire et meurtrière. Dans les textes de la tradition judéohellénistique, la mouche (muia) est également considérée comme un insecte meurtrier. C'est par exemple le cas de Sg 16,9, où elle est jumelée à la sauterelle. Dans De Vita Mosis i 130, Philon d'Alexandrie présente les mouches (muias) et le chien comme étant les bêtes les plus effrontées, les plus insatiables de butin (anaides). Quant à la mouche, elle est non seulement la plus hardie (thrasutatos) de tout ce qui vole, mais elle est également décrite à l'aide de verbes qui ont des connotations militaires: elle vient vers son ennemi (le verbe epiphoitaõ signifie « faire une incursion dans un territoire ennemi »), elle se jette sur lui pour l'attaquer (epitrechõ) et elle est invincible (aettetos) (Philon 1967, 86).

4. Par contre, Symmaque a rendu l'expression zbwby mwt par muiõn thanatos, «la mort des mouches ", inversant ainsi le sens des mots du texte hébraïque. Pour les textes de Symmaque, Aquila et Théodotion, nous travaillons à partir de l'édition des Hexaples d'Origène $(1863,1553)$.

5. Il s'agit d'une déformation méprisante de Baal Zeboul, Baal le Prince, ou Baal de la demeure (céleste), divinité cananéenne particulièrement vénérée à Eqrôn. Dans le Nouveau Testament, le mot désigne désormais un démon, voire le chef des démons (Mt 10,25; 12,24.27; Mc 3,22; Lc 11,15.18.19). 
En définitive, s'il est vrai que l'expression zbwby mwt est ambiguë, la traduction "mouches meurtrières" est justifiée du point de vue grammatical et du point de vue de l'analyse comparée. Qui plus est, cette traduction a l'avantage de montrer que l'expression $z b w b y$ mwt appartient au riche vocabulaire politico-militaire qu'on retrouve dans le contexte immé-

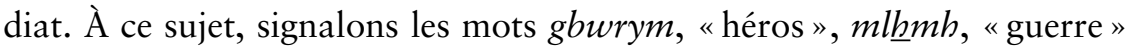
$(9,11) ; m l k$, « roi », sbb, « encercler », bnh ' $l$, « construire contre », mtswdym, «embuscades » $(9,14) ;$ gbwrh, « force » $(9,16) ;$ mwšl, «chef » $(9,17 ; 10,4)$; et $k l y$ qrb, «armes de guerre» $(9,18)$.

Nous avons déjà noté que le verbe $y b$ 'yš, qui se rattache à l'expression zbwby mwt, est au singulier. Quelques exégètes expliquent qu'il s'agit d'un singulier distributif qui fait référence à « chaque mouche» (Rottzoll 1999, 207-208; Delsman 1991, 35). Par ailleurs, il est bien connu que les noms pluriels de choses ou d'animaux peuvent être considérés comme équivalant à des collectifs; le verbe se met alors au singulier (voir, par exemple, Jl 1,20; Is 59,12; etc.; Joüon 1982, 460). Qui plus est, comme une telle absence d'accord entre le verbe et le sujet n'est pas inusitée dans le livre de Qo $(1,10.16 ; 2,7)$, il n'y a aucune raison valable de corriger le verbe $y b$ ' $y$ š. Cependant, la Bible de Jérusalem n'est pas de cet avis, puisqu'elle suppose que le texte hébreu a été mal coupé: "litt. "sont la cause" yabi’û sheyabbi'a". Elle traduit donc le premier stique comme suit: "Des mouches mortes font que le parfumeur rejette l'huile» $(1998,1109)$. Une telle traduction n'a aucun appui textuel en sa faveur et occulte le contraste entre la puanteur et l'huile du parfumeur. De la même façon, la traduction par «ruiner » proposée par L'hour et al. $(2001,1660)$ n'est pas plus heureuse, car elle souligne moins bien le contraste avec l'huile du parfumeur qui apparaît à la fin du stique. En effet, le verbe $b$ 'š signifie clairement «puer» et comme il est employé au mode hiphil, il signifie littéralement «rendre puant », "empuantir ", « empester».

Le mot qui suit, $y b y^{\varsigma}$, dérive de la racine $n b^{\varsigma}$, « jaillir». Ce verbe est également au mode hiphil, le causatif, et devrait donc être littéralement traduit

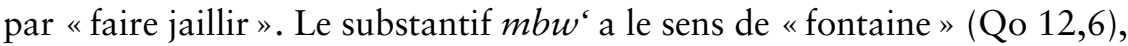
tandis que l'expression mbw'y mym désigne les « eaux jaillissantes » (Is 35,7; 49,10). Quant au verbe $n b^{\prime}$, il renvoie une fois à l'image du «fleuve jaillissant » $(\operatorname{Pr} 18,4)$. Dans tous les autres cas, il est employé métaphoriquement pour désigner le flot de paroles (Ps 19,3 ; 59,8 ; 78,2 ; 94,4 ; 119,171; $145,7 ; \operatorname{Pr} 15,8)$ ou le souffle de la sagesse (Pr 1,23). Ibn Ezra donne un sens transitif au verbe $y b y^{\prime}$ et le rattache à la mouche: la puanteur ne cesse, car la 
mouche continue de barboter et de couler dans l'huile (Rottzoll 1999, 208). Cette dernière interprétation est invraisemblable, car elle suppose qu'on donne deux sens différents à deux verbes qui sont au mode hiphil et qui se suivent.

Comme la signification première de ce verbe ne fait guère de sens dans le présent contexte et puisqu'il est juxtaposé au verbe précédent sans conjonction $w$, d'aucuns y voient une dittographie et proposent donc de l'éliminer (Strobel 1967, 148; Galling 1969, 116; Zimmerli 1980, 225). Le verbe avait déjà posé des difficultés aux anciens traducteurs et c'est sans doute pourquoi Symmaque et Jérôme, aussi bien dans la Vulgate que dans son commentaire, ont omis de le traduire. Toutefois, comme toutes les autres versions anciennes supposent l'existence d'un mot entre $y b^{\prime} y \check{s}$ et šmn, l'éliminer ne constitue pas une solution raisonnable. Certains y voient plutôt une glose (Lauha 1978, 179-180; Whybray 1989, 150), mais cette hypothèse ne règle rien. Au contraire, si l'on en juge d'après les commentateurs, loin de clarifier le texte, le soi-disant glossateur l'aurait plutôt obscurci!

La LXX a rendu le verbe $y b y^{\prime}$ par un substantif qui est un hapax: skenasia, "préparation». De son côté, peut-être sous l'influence de la Lxx, la Peshitta a traduit le verbe $y b y^{\prime}$ par le substantif $m^{\prime} n^{\prime}$, "vase ». Il est également possible qu'elle ait repris ce mot de 9,18 où il apparaît au pluriel au sens de «instruments».

À l'instar de la Lxx, de la Peshitta et de la Syro-Hexaplaire qui a le mot twqn', "récipient ", d'aucuns préfèrent corriger le mot et lire $g^{e} b \hat{\imath}^{\natural} a$, "calice», «coupe » (Sacchi 2005, 204; Frydrych 2002, 190; Fox 1999, 301; Seow 1997, 312). Pour justifier cette correction, Fox fait remarquer que dans l'écriture ancienne (par exemple, 1QIsa), le trait du yod se prolonge tellement vers le bas qu'il finit par ressembler au gimel. Cette lecture, ajoute$\mathrm{t}$-il, règle le problème du lien asyndétique quelque peu difficile des deux verbes du texte massorétique. Par contre, une telle correction n'a aucun appui textuel, ni dans les anciens manuscrits hébraïques, ni dans les traductions grecques (Goldman 2004, 105). Le mot grec skeuasian, en effet, n'est aucunement l'équivalent du mot $g^{e} b \hat{\imath}^{\prime} a$, pas plus d'ailleurs que le mot skeuates ou skeuos, car le mot $g^{e} b \hat{\imath}^{\prime} a$ est traduit par kondu (Gn 44,2.12.16.17), krater (Ex 25,31.33.34) et keramion (Jr 42,5). La leçon la plus difficile, soit celle du texte massorétique, semble donc la plus ancienne. Rose estime également qu'il n'est pas nécessaire de modifier le texte hébreu. Selon lui, il faut simplement considérer la forme consonantique $y b^{\circ}$ comme 
un nom avec la préformante yod (comme, par exemple, yits $\underline{e} \underline{\text { a } r}$, " huile») qui indique l'état fluide de la préparation (skeuasia) d'une huile purifiée, une décoction (1999, 416-417). Cette hypothèse est inutilement alambiquée.

Driver fait appel à la racine arabe $n b g h$, « jaillir, bouillir, fermenter »,

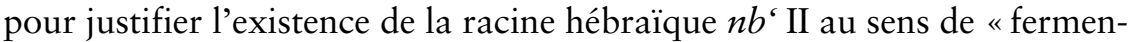
ter» (1954, 231-232). Schoors juge qu'il n'est pas acceptable de choisir la signification rare d'une racine arabe afin de prouver que la même racine existe en hébreu avec un sens identique. Qui plus est, selon lui, comme la racine $n b g h$ signifie normalement " surgir, émerger, ressortir, exceller ", elle ne suffit aucunement à prouver l'existence d'une racine hébraïque signifiant «fermenter» $(2004,402)$. Quoi qu'il en soit de l'analyse philologique comparée, plusieurs exégètes traduisent le verbe $y b y^{\prime}$ par «faire fermenter» (Delsman 1991, 35; Krüger 1997a, 63 ; Brandscheidt 1999, 194; Schwienhorst-Schönberger 2004, 481) ou "faire tourner» (Kahn 1989, 1050), estimant, d'une part, qu'il s'agit là d'une extension raisonnable du

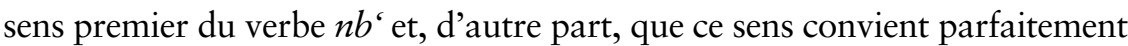
au contexte du v. 1 .

Geier en fait un auxiliaire du verbe $b$ 'š et traduit les deux verbes par «faire puer en permanence» (Schoors 2004, 401). Dans un sens semblable, Shields interprète l'expression $y b^{\prime} y$ '̌ $^{\prime} y b y^{\prime}$ comme un hendiadys verbal asyndétique qui souligne la nature exubérante de l'odeur, d'où la traduction "dégager une odeur infecte» $(2006,210)$. En résumé, l'explication selon laquelle le verbe «faire fermenter » ou «infecter » est une extension raison-

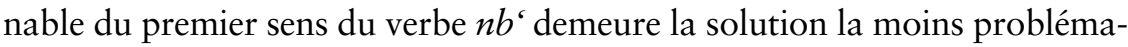
tique et la plus fidèle au texte massorétique. Cette explication a aussi l'avantage de considérer les deux verbes comme des synonymes, lesquels constituent peut-être, par le fait même, un hendiadys verbal asyndétique qui souligne la gravité de la corruption causée par les zbwby mwt.

Le mot šmn, littéralement "huile », ne pose aucune difficulté textuelle. Assez curieusement, aucun exégète n'a remarqué que l'huile est ici un symbole de bonheur, de réjouissance et de fête. C'est également très clair en 9,8, mais aussi en 7,1 (Lavoie 1998, 100-101), les deux seuls autres passages où le mot apparaît dans le livre de Qo. Maints autres textes de la Bible attestent aussi que l'huile est un symbole de joie et de bonheur (voir 2 S 12,20; Am 6,6; Ps 23,5; Pr 27,9; Jud 10,3; Sg 2,7; etc.). C'est d'ailleurs ce que confirme le dernier mot du stique, le qal participe $r w q \underline{h}$, "préparateur de parfum ", qui n'apparaît qu'ici en Qo. Au sujet de ce dernier mot, Rose affirme que la leçon de la Lxx (elaiou hedusmatos, «huile d'agrément») 
suppose la vocalisation rôqa $\underline{h}$, au lieu du participe rôqe $\underline{h} a(1999,417)$. Il en déduit que le mot $r w q \underline{h}$ désigne l'excellence de cette huile. Cette hypothèse est peu probable, car le mot hedusma ne traduit jamais la racine rqh dans la Lxx. Qui plus est, le mot rôqah n'apparaît jamais dans la Bible, tandis que le mot rôqeha est attesté à six autres reprises (Ex 30,25.35; $37,29 ; 1 \mathrm{Chr} 9,30$; Sir 38,7; 49,1 $)^{6}$. Il est vrai que la Peshitta rend le mot rwqh par bsnm', "agréable ", "douce », mais c'est parce qu'elle suit ici le texte grec. La même remarque s'impose pour les traductions de Jérôme: suavitatem unguenti, "suaveté d'un parfum »(Vulgate) et oleum compositionis, «huile de composition (de parfum)» $(1959,332)$. De son côté, Symmaque suppose bel et bien le participe rôqeha du texte massorétique, car il traduit l'expression šmn rwqh par elaion euõdes murepsou, « huile agréable de parfumeur» (Origène 1863, 1553). Par ailleurs, en employant le mot euõdes, qui est synonyme du mot hedusma qu'on retrouve dans la Lxx, il traduit un mot absent du texte massorétique. Or, comme le mot bšm, "parfum », "bonne odeur », a été traduit à la fois par euõdes (Ex 30,23) et hedusma (1 R 10,2.10.25; Ez 27,22), faut-il supposer que ce mot faisait originellement partie du texte massorétique et qu'il devait se lire à la suite du mot šmn? Goldman est fortement tenté par cette hypothèse, mais il reconnaît qu'il n'est pas facile de reconstituer l'histoire du texte (2004, 105-106). À notre avis, cette hypothèse est peu vraisemblable, car la leçon la plus courte est habituellement la plus ancienne. C'est pourquoi nous maintenons le texte massorétique. En définitive, Qo 10,1a fait donc allusion au bonheur, symbolisé par l'huile du parfumeur, qui est corrompu par le pouvoir militaire, symbolisé par les mouches meurtrières.

Notre interprétation est également confirmée par Qo 9,18b qui introduit la seconde critique de la valorisation de la sagesse et dont le v. 1a constitue une illustration complémentaire. Voici notre traduction de ce v. 18: "Mieux vaut la sagesse que les armes de guerre, mais un seul gaffeur détruit beaucoup de bonheur. » Seuls deux mots font ici l'objet d'interprétations divergentes. Le premier est le mot $\underline{h} w \underline{t}$, que de rares commentateurs traduisent encore par "pécheur» (Eaton 1983, 132; Shields 2006, 208). De nos jours, en effet, les exégètes sont presque unanimes à reconnaître que le mot $\underline{h} w \underline{t}$, dont le sens premier est «manquer le but " (Jg 20,16; Is 45,20; $\operatorname{Pr} 8,36 ; 10,16 ; 19,2 ; 20,2$; Jb 5,24; etc.), ne présente ici aucune connotation morale, d'où les traductions par «gaffeur ", "raté ", "maladroit ",

6. L'expression šmn rwq $\underline{h}$, "huile de parfumeur ", est même attestée à Ougarit (Dahood $1952,212 ; 1966,278)$. 
etc. (Gordis 1968, 310; Glasser 1970, 148; Fox 1999, 300 ; etc. ${ }^{7}$ ). Qui plus est, le présent contexte indique bien que le gaffeur est ici un homme politique, comme le chef du v. 17, car il a le pouvoir de détruire beaucoup de $t w b h$. Tel est le second mot qui suscite diverses interprétations. Bien que les exégètes reconnaissent presque tous que le mot $\underline{h w} \underline{t}$ ' n'a ici aucune connotation morale, d'aucuns traduisent quand même le mot twbh par "bien» (Traduction oecuménique de la Bible 1975, 1630; Bible de Jérusalem 1998, 1109), pouvant ainsi laisser entendre qu'il a une connotation morale. Par ailleurs, Ogden estime que twbh est ici l'équivalent de la sagesse et de ses bénéfices $(1980,163)$. Selon Krüger, le mot twbh en 9,18b désigne plutôt la richesse matérielle (1997b, 55). À notre avis, le mot n'exclut pas les richesses matérielles, mais il désigne encore plus globalement le bonheur $^{8}$, lequel est toujours très concret dans le livre de Qo9 . En résumé, 10,1a poursuit le même objectif que $9,18 \mathrm{~b}$, mais en employant un langage symbolique: de même qu'un seul gaffeur politique détruit beaucoup de bonheur, ainsi des mouches meurtrières (symbole du pouvoir militaire) corrompent l'huile du parfumeur (symbole du bonheur).

\section{Qobélet 10,1b}

Le texte massorétique du v. $1 \mathrm{~b}$ se lit comme suit:

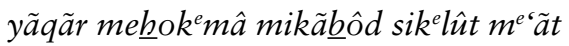

La leçon de la Lxx est plutôt surprenante. Si tous les mots ont été rendus correctement, à l'exception de l'omission du $m$ devant $\underline{h} k m b$ et de l'ajout de l'adjectif megas, la traduction s'écarte carrément du texte massorétique: timion oligon sophias huper doxan aphrosunes megales, "précieuse (chose) qu'un peu de sagesse, plus que la gloire d'une grande folie »,

7. Il est inutile de suivre les exégètes comme Loader $(1979,60)$, Sacchi $(2005,203)$ et Rose (1999, 412), qui proposent de corriger bôt $\underline{\hat{e}}$ ' en hete', lequel mot devrait alors être rendu non pas par "péché ", mais bien par "erreur" (ainsi Loader et Sacchi), «manquement» (ainsi Rose) ou "maladresse », "gaffe ", etc. Il est vrai que la version syriaque appuie une telle correction. Toutefois, à l'instar des meilleurs témoins du texte tibérien, comme l'atteste le codex Leningradensis, la Lxx et le Targum supposent bel et bien l'hébreu $\underline{h} \hat{o} \underline{t} \hat{e}$ '.

8. Comme en Qo 2,1.3.24; 3,12.13.22; 5,17; 6,3.6; 7,14[2x]; 8,12.13.15; 11,7.

9. C'est du moins ce que montrent des mots clés comme manger et boire (' $k l$ et $\mathrm{s}^{\prime} t h$ : 2,1-3.24-26; 3,12-13 ; 5,17-18; 8,15; 9,7; 10,19), goûter ( $r^{\prime} h: 2,1.24 ; 3,13 ; 5,17 ; 6,6$; 9,9), pain (lhm:9,7), vin (yyn:2,3;9,7;10,19), vêtements blancs (bgdy lbnym:9,8) et huile $(\check{s} m n: 7,1 ; 9,8)$. 
ce qui suppose le texte suivant: yqr hkmh m't mkbwd sklwt rbh. En faisant en sorte que le mot « peu » qualifie la «sagesse » et non la "folie », le traducteur a réussi à édulcorer la conclusion ironique et choquante du texte massorétique. Il s'agit ici manifestement d'une paraphrase moralisatrice.

La version syriaque est ici plus proche du texte massorétique que du texte grec: yqyr' $m n \underline{h} k m t^{\prime}$ ' $w m n$ swg't tšbwhth' sklwt' qll, "plus précieuse / plus lourde que la sagesse et que beaucoup de gloire un peu de folie ». Le mot yqr' est ici nettement ambigu: en syriaque, contrairement à l'hébreu biblique, il peut aussi bien désigner ce qui est précieux que ce qui est lourd (Payne Smith 1967, 196; Costaz 2002, 144). Par ailleurs, au lieu de qll, "peu », quelques manuscrits ( $8 \mathrm{aI}^{1}, 9 \mathrm{cI}, 10 \mathrm{cI}, 11 \mathrm{cI}, 12 \mathrm{aI}{ }^{\mathrm{mg}}$ ) ont le mot $r b t^{\text {', }}$ "beaucoup »: " beaucoup de folie ». Il s'agit vraisemblablement d'une correction effectuée à la suite de la traduction aphrosunes megales, "grande folie ", proposée par la LXx.

Du côté des traducteurs modernes, un seul mot a fait l'objet de modifications. Certains proposent, en effet, de corriger $m k b w d$ en $m^{\prime} b d$ ou m’byd (ainsi Siegfried, Zapetal et Galling, cités dans Krüger 1997a, 62), du verbe ' $b d$, «détruire ", supposant ainsi qu'il suffit d'un peu de folie pour détruire la sagesse. Au lieu de remplacer le kaph par un 'aleph, Rose suggère de le remplacer par un $\underline{h} \hat{e} \underline{t}$, lisant ainsi le verbe $k \underline{h} d$ au mode hiphil, au sens de «cacher, faire disparaître » : " un peu de sottise suffit pour enlever à la sagesse toute sa valeur » (1999, 415 et 418). Quelques exégètes préfèrent lire mkbyr, participe hiphil du verbe $k b r$, «multiplier ", d'où les traductions suivantes: "le quart d'un sou de folie est plus précieux que la sagesse en surabondance » (Strobel 1967, 148); « et un peu de folie abonde sur la sagesse " (Frydrych 2002, 190). Enfin, dans la première version de son commentaire, Fox identifie le mot yqr comme une épithète de šmn $r q \underline{b}$ et corrige le mot $m k b w d$ en $t k b d$, ce qui donne la traduction suivante: " un peu de folie pèse plus que sagesse» $(1989,261$ et 265). Ces diverses corrections de mkbwd sont toutes inacceptables, car elles n'ont aucun appui textuel. Il est également inutile d'y voir une glose (Whybray 1989, 150), car celle-ci n'éclaire en rien le sens du texte. La seule variante textuelle attestée par plusieurs manuscrits hébraïques et adoptée par de rares traducteurs (par exemple, Willmes 2000, 218) est l'ajout d'un $w$ devant $m k b w d$, ce qui rend la formulation asyndétique moins difficile.

Gordis donne au mot kbwd le sens d' "abondance » : "plus que sagesse en abondance ». Pour justifier sa traduction, il cite Is 10,3 où le mot kbwd a le sens de «richesse» et tout particulièrement Is 5,13 où le mot signifie 
«multitude» $(1968,315)$. Cette traduction, précise-t-il, a l'avantage de bien souligner le contraste entre l'abondance de sagesse et le peu de folie. Outre le fait que la traduction qu'il propose du mot kbwd en Is 5,13 est fortement discutable ${ }^{10}$, cette interprétation n'explique pas la présence du $m$ qui précède le mot kbwd et encore moins la présence du $w$ dans maints manuscrits. Tout autre est le point de vue de Glasser. Il donne au mot kbwd son premier sens, soit celui de poids, et traduit le $m$ qui le précède par "sans ", d'où sa traduction littérale "sans poids ", c'est-à-dire impossible à peser. Il en conclut que l'expression $m \underline{h} k m b m k b w d$ doit être rendue comme suit: «plus que sagesse inestimable»(Glasser 1970, 148). Bien qu'ingénieuse, cette interprétation a le désavantage de donner deux sens différents aux deux emplois de la préposition $m$ qui se succèdent. Pour le mot $k b w d$, il vaut donc mieux maintenir son sens le plus usuel, soit celui de «gloire » ou d' « honneur ", comme c'est le cas en 6,2, qui est le seul autre passage du livre où le mot revient. Quant aux emplois de la préposition $m$ devant les mots $\underline{h} k m b$ et $k b w d$, ils ont un sens comparatif et expriment une différence en plus (Joüon 1982, 406 et 435-436).

Pour bien saisir la portée de la comparaison, il faut savoir comment traduire le mot yqr. Les uns lui donnent le sens qu'il a en araméen ou en hébreu post-biblique, soit celui de «lourd ( Loader 1979, 60; Seow 1997, 312; Fox 1999, 302 ; Schoors 2004, 388; Forti 2005, 244-245), d'où la traduction littérale suivante: «lourde plus que sagesse, que gloire, un peu de folie ". Autrement dit, la lourdeur de la folie a une signification dépréciative: il suffit d'un peu de folie (sklwt $\left.m^{\prime} t\right)$ pour mettre en échec sagesse $(\underline{h} k m h)$ et gloire $(k b w d)$, tout comme une ou des mouches suffisent pour empester une huile de parfumeur. En résumé, 10,1 ne fait que confirmer ce qui a déjà été dit en Qo 9,18b, à savoir que la sagesse est vulnérable et que sa valeur est donc relative (Ogden 1980, 32; D’Alario 1993, 159-160; Sayler 2001, 362).

Cette interprétation pose toutefois au moins deux problèmes. Premièrement, il n'y a aucun terme dans le texte hébreu qui permette de croire que le v. $1 \mathrm{~b}$ doive nécessairement se comprendre comme une simple analogie du v. 1a. Par conséquent, en introduisant le v. 1b par «ainsi » (Garrett 1993, 333 ; Forti 2005, 244 ; Shields 2006, 208) ou "par analogie»

10. Dans ce texte, en effet, le mot $k b w d w$ doit plutôt être rendu par «sa noblesse» ou "son élite », car il forme avec le mot hmwnw, «sa multitude », qui apparaît en parallélisme, un couple de totalité qui représente les deux classes du peuple (' $m$ ) évoqué au v. $13 \mathrm{a}$. 
(Ogden 1980, 32), les exégètes paraphrasent plus qu'ils ne traduisent. Deuxièmement, la traduction de yqr par «lourd » est incorrecte, car ce mot n'a jamais cette signification dans le reste de la Bible hébraïque. Il a toujours une connotation positive et désigne ce qui est précieux, ce qui a de la valeur (Krüger 1997a, 64) ${ }^{11}$. C'est aussi ce que confirme la Lxx qui n'a jamais rendu la racine yqr par des mots qui renvoient à l'idée de lourdeur. Au contraire, la racine $y q r$ a toujours été rendue par des mots qui signifient ce qui est précieux (timios), ce qui a de la valeur (time), ce qui est estimé ou honoré (entimos), etc. (Muraoka 1998, 64). Qui plus est, même dans les textes de Qumran, le mot yqr n'a jamais la signification de «lourd ». Par exemple, le mot y désigne ce qui est précieux (4Q 504 1-2iv10), magnifique (4Q 171 iii5a), merveilleux (4Q 405 15ii-16,6), honorable (4Q 286 1ii1), etc. (Abegg et al. 2003, 320). Bref, nous croyons que le second stique doit plutôt se traduire comme suit: "plus précieuse que sagesse, que gloire, un peu de folie». Par ailleurs, les exégètes qui optent pour cette traduction de $y q r$ ne partagent pas forcément la même interprétation. Selon Lauha, cette affirmation est ironique: elle souligne que le pouvoir de la sagesse est fragile, d'autant plus qu'elle est une réalité totalement profane, qui n'a aucune dimension divine (1978, 181-182). Indépendamment de Lauha, Backhaus est également d'avis que le mot yqr, "précieuse ", a une valeur ironique et que cette ironie joue une fonction critique, celle de rappeler les limites de la sagesse (2000, 43). Willmes abonde dans le même sens (2000, 219). Pour sa part, Krüger n'y voit aucune ironie. Selon lui, le v. 1b dégage la conclusion des images qui viennent d'illustrer la faiblesse de la sagesse: si l'efficacité de la sagesse dépend de sa reconnaissance sociale, alors, aussi peu que possible, la folie (de la majorité de la population) est plus précieuse que la sagesse et l'honneur (d'une petite élite). Il en déduit que l'évaluation réaliste des forces et des faiblesses de la sagesse, telle qu'elle est présentée en 9,13-10,1, soutient un programme d'éducation populaire au lieu d'une éducation réservée à une minorité élitiste (1997a, 69, 74-75 et 2004, 180). Strobel, dont on a déjà vu qu'il corrige mkbwd en lmkbyr, propose une interprétation totalement différente. Selon lui, en 10,1b, Qo a recours à un proverbe qui enseigne la voie du juste milieu $(1967,149)$. Bien qu'il ignore ce dernier commentaire, Klein propose une interprétation

11. Telle est aussi la traduction adoptée par Strobel $(1967,148)$, Lauha $(1978,179$ et 181), Klein (1994, 78), Brandscheit $(1999,194)$ et Backhaus $(2000,42)$. 
semblable, mais en maintenant le mot kbwd au sens de «honneur» (1994, 78-79 et 149). Selon lui, en reconnaissant que la sagesse qui est accompagnée d'un peu de folie est bonne $(9,18$ a et $10,1 \mathrm{~b})$, Qo recommande la voie du milieu (voir 6-18), qui se trouve entre les deux positions extrêmes, c'està-dire entre celle qui affirme que la sagesse est bonne bien qu'elle soit souvent inutile $(9,13-17)$ et celle qui reconnaît que la pure folie n'est jamais valorisée puisqu'elle cause des dommages $(10,1 \mathrm{a} ; 9,18 \mathrm{~b} ; 10,2-3)$. Pour justifier son interprétation, Klein réorganise l'ensemble des stiques de 9,1810,1 comme suit: 9,18a; 10,1b;10,1a ; 9,18b;10,2-3. Or, comme le remarque Krüger, cette réorganisation est parfaitement arbitraire (1997a, 64). Elle l'est d'autant plus que nous avons bien montré que 9,17-10,1 est un passage structuré en chiasme autour d'une double opposition: d'une part, entre une comparaison qui affirme la supériorité de la sagesse $(A: 9,17$ : comparaison en "plus que ») et une autre qui affirme la supériorité de la folie ( $\mathrm{A}^{\prime}: 10,1 \mathrm{~b}$ : comparaison en "plus que ») et, d'autre part, entre un dit de préférence qui valorise la sagesse (B: 9,18a: comparaison en "mieux vaut ») et un commentaire critique introduit par «mais ", qui illustre sa valeur relative en soulignant la précarité du bonheur (B': 9,18b-10,1a).

Toutefois, l'interprétation de Klein n'est pas complètement erronée. Qo 10,1b ne fait pas que souligner la limite ou la vulnérabilité de la sagesse. La conclusion de 10,1b est encore plus audacieuse, puisqu'elle valorise, non sans ironie, la folie, mais sans excès. Cette valorisation de la folie en petite quantité n'est pas totalement surprenante, car la quête de Qo n'a pas exclusivement porté sur la sagesse, mais aussi sur la folie. Qohélet, en effet, a «dû appliquer son cœur à comprendre la sagesse et à comprendre la démence et la folie $(\breve{k} k l w t) »(1,17 \mathrm{a})$. Qui plus est, afin de ne pas manquer une seule occasion de bonheur, il s'est également attaché à la folie (sklwt) jusqu'à ce qu'il voit si c'est là le bonheur pour les fils d'Adam qui s'affairent sous le ciel $(2,3 \mathrm{c})$. Puis, après avoir de nouveau considéré la sagesse, la démence et la folie (sklwt) $(2,12)$, il est vrai qu'il affirme qu'il y a profit de la sagesse sur la folie $(2,13)$, mais il s'empresse aussitôt de relativiser ce profit en rappelant que la mort scelle de manière identique le sort du sage et de l'insensé $(2,14 b-16)$. Son verdict final est alors tout aussi surprenant que celui qu'il émet en $10,1 \mathrm{~b}$, puisqu'il conclut qu'il déteste la vie $(2,17$; comparer, par exemple, à 9,4 et à ses nombreuses invitations à jouir de la vie!). C'est sans doute pourquoi, en 7,16-17, il rappelle avec ironie que ni la sagesse ni la folie ne garantissent la sécurité. Cette invitation à ne pas 
commettre d'excès explique sans doute la présence du mot $m^{\prime} t$, "peu »: seule la folie à petite dose $\left(s k l w t m^{\prime} t\right)$ est plus précieuse que sagesse et gloire.

\section{Conclusion}

Qo 10,1 est un verset énigmatique parce qu'il pose des difficultés du point de vue textuel, sémantique, structurel et référentiel. Du point de vue textuel, nous avons montré qu'aucune correction ne s'impose. Quant aux modifications apportées par les anciennes versions, elles proviennent soit du fait que certaines racines verbales ont été mal comprises (par exemple, en 10,1a de la Lxx, de la Vulgate et de la Peshitta), soit du fait que le texte hébreu est apparu trop choquant et qu'il fallait l'édulcorer (par exemple, en 10,1b de la LXX). Du point de vue sémantique, l'analyse de chacun des mots de 10,1 nous a permis de justifier la traduction suivante: "Des mouches meurtrières empestent, infectent une huile de parfumeur; plus précieuse que sagesse, que gloire, un peu de folie.» Quant au texte de 9,17-18, il se lit comme suit: «Les paroles des sages écoutées dans le calme valent mieux que les cris d'un chef au milieu des insensés. Mieux vaut la sagesse que les armes de guerre, mais un seul gaffeur détruit beaucoup de bonheur. » Du point de vue sémantique et structurel, nous avons également relevé des indices qui indiquent que $9,18 \mathrm{~b}-10,1 \mathrm{a}$ traite du même thème et que ces deux stiques s'opposent à 9,18a, tandis que 10,1 b s'oppose à 9,17. Du point de vue référentiel, nous avons présenté de bons arguments qui permettent de conclure que l'image des «mouches meurtrières » n'est pas étrangère au riche vocabulaire politico-militaire qu'on retrouve dans le contexte immédiat. En ce qui concerne l'huile du parfumeur, elle symbolise clairement le bonheur (voir 7,1 et 9,8). En résumé, nous avons démontré que Qo 10,1 propose deux commentaires critiques qui viennent relativiser la valeur traditionnelle de la sagesse:

1) En évoquant le monde politico-militaire qui a le pouvoir de détruire le bonheur, 9,18b-10,1a s'oppose à 9,18a qui affirme la supériorité de la sagesse sur les armes;

2) En soulignant qu'un peu de folie a plus de valeur que la sagesse et la gloire, 10,1b s'oppose à 9,17 qui affirme également sa préférence pour la sagesse. Le mot clé est ici $m$ 't, "peu » : la valorisation de la folie au détriment de la sagesse et de la gloire n'est possible que parce que celle-ci n'est pas excessive. En effet, celui-là même qui, dans sa quête de bonheur, s'est intensément intéressé aussi bien à la 
sagesse qu'à la folie (voir 1,$17 ; 2,3$ ) est également celui qui rappelle, non sans ironie, que ni la sagesse ni la folie ne garantissent le bonheur et la sécurité (voir 7,16-17).

\section{Références}

Abegg, M. et al., (2003), The Dead Sea Scrolls Concordance. Volume One. The Non Biblical Texts from Qumran, Leiden, Brill.

BackHaus, F. J. (2000), "Kohelet und die Ironie. Vom Umgang mit Widersprüchen durch die Kunst der Ironie ", Biblische Notizen, 101, p. 29-55.

Bible de Jérusalem (1998), Paris, Cerf.

Biblia Sacra iuxta latinam Vulgatam versionem (1957), Romae, Typis Polyglottis Vaticanis.

BRANDSCHEIDT, R. (1999), Weltbegeisterung und Offenbarungsglaube. Literar-,form- und traditionsgeschichtliche Untersuchung zum Buch Kohelet, Trier, Paulinus (Trierer Theologische Studien, 64).

Costaz, L. (2002), Dictionnaire syriaque-français, Beyrouth, Dar elMachreq.

DAHOOD, M. (1952), "Canaanite-Phoenician Influence in Qoheleth », Biblica 33, p. 30-52.

(1966), «The Phoenician Background of Qoheleth », Biblica, 47, p. $264-282$.

D'Alario, V. (1993), Il libro del Qohelet. Struttura letteraria e retorica, Bologna, Éd. Dehoniane (Supplementi alla Rivista Biblica, 27).

Delsman, W. C. (1991), "Die Inkongruenz im Buch Qohelet», dans K. Jongeling et al., dir., Studies in Hebrew and Aramaic Syntax. Presented to Professor J. Hoftijzer, Leiden, Brill, p. 27-37.

Driver, G. R. (1954), «Problems and Solutions », Vetus Testamentum, 4, p. 225-245.

Eaton, M. A. (1983), Ecclesiastes. An Introduction and Commentary, Illinois, Inter-Varsity Press (The Tyndale Old Testament Commentaries, $16)$. 
FORTI, T. (2005), «The Fly and the Dog: Observations on Ideational Polarity in the Book of Qoheleth ", dans R. L. Troxel et al., dir., Seeking Out the Wisdom of the Ancients. Essays Offered to Honor Michael V. Fox on the Occasion of his sixty-fifth Birthday, Winona Lake, Eisenbrauns, p. 235255 .

Fox, M. V. (1989), Qohelet and his Contradictions, Sheffield, The Almond Press (Journal for the Study of the Old Testament. Supplement, 71).

(1999), A Time to Tear Down \& a Time to Build Up. A Rereading of Ecclesiastes, Grand Rapids, Eerdmans.

Frydrych, T. (2002), Living under the Sun. Examination of Proverbs and Qoheleth, Leiden, Brill (Vetus Testamentum Supplements, XC).

Galling, K. (1969), Der Prediger, Tübingen, J.C.B. Mohr (Handkommentar zum Alten Testament, 1,18).

Garrett, D. A. (1993), Proverbs. Ecclesiastes. Song of Songs, Nashville, Broadman Press (The New American Commentary, 14).

Glasser, É. (1970), Le procès du bonheur par Qohélet, Paris, Cerf (Lectio Divina, 61).

Goldman, Y. A. P. (2004), "Qoheleth", Biblia Hebraica Quinta editione cum apparatu critico novis curis eleborato. General Introduction and Megilloth, Stuttgart, Deutsche Bibelgesellschaft, p. 25-53 et 64-112.

Gordis, R. $\left(1968^{3}\right)$, Koheleth. The Man and his World, New York, Schocken Books.

Jérôme (1959), S. Hieronymi Presbyteri Opera. Pars I,1, Turnholti, Brepols (CCSL, 72).

JoÜON, P. (1982), Grammaire de l'hébreu biblique, Rome, Institut biblique pontifical.

KAHN, Z. (19897), dir., La Bible traduite du texte original par les membres du rabbinat français, Paris, Colbo.

KLEIN, C. (1994), Kohelet und die Weisheit Israels. Eine formgeschichtliche Studie, Stuttgart, Kohlhammer (Beiträge zur Wissenschaft vom Alten und Neuen Testament, 132).

KRÜGER, T. (1997a), "Wertvoller als Weisheit und Ehre ist wenig Torheit" (Kohelet 10,1) », Biblische Notizen, 89, p. 62-75.

(1997b), «Das Gute und Güter. Erwägungen zur Bedeutung von twb und twbh im Qoheletbuch", Theologische Zeitschrift, 53, p. 53-63. 
(2004), Qoheleth: A Commentary, Minneapolis, Fortress Press (Hermeneia).

Lamparter, H. (1955), Das Buch der Weisheit. Prediger und Sprüche, Stuttgart, Calwer Verlag (Die Botschaft des Alten Testament, 16,1).

Lane, D. J. (1979), The Old Testament in Syriac According to the Peshitta Version, Part II, Fascicule 5, Leiden, Brill.

LauHA, A. (1978), Kohelet, Neukirchen, Neukirchener Verlag (Biblischer Kommentar. Altes Testament, 19).

LAvoIE, J.-J. (1998), «La philosophie comme réflexion sur la mort. Étude de Qohélet 7,1-4 », Laval théologique et philosophique, 54, p. 91-107.

L'Hour, J. et al. (2001), "Paroles de Qohélet », dans La Bible. Nouvelle traduction, Paris/Montréal, Bayard/Médiaspaul, p. 1633-1666.

LOADER, J. A. (1979), Polar Structures in the Book of Qohelet, Berlin, Walter de Gruyter (Beihefte zur Zeitschrift für die alttestamentliche Wissenschaft, 152).

LoAder, J. A. (1986), Ecclesiastes. A Practical Commentary, Grand Rapids, Eerdmans.

Muraoka, T. (1998), Hebrew / Aramaic Index to the Septuagint Keyed to the Hatch-Redpath Concordance, Grand Rapids, Baker Book.

Ogden, G. S. (1980), "Qoheleth Ix 17-x 20. Variations on the Theme of Wisdom's Strength and Vulnerability ", Vetus Testamentum, 30, p. 27-37.

OrigÈne (1863), "Hexaples », dans Patrologie grecque 16, Paris, Migne, p. $1493-1570$.

Payne Smith, J. (1967), A Compendious Syriac Dictionary Founded Upon the Thesaurus Syriacus of R. Payne Smith, Oxford, Clarendon Press.

Philon D’AleXandrie (1967), De Vita Mosis I-II. Intro., trad. et notes par R. Arnadelz, et al. Paris, Cerf.

RaHLFs, A. (1935), Septuaginta, Stuttgart, Deutsche Bibelstiftung.

Rose, M. (1999), Rien de nouveau. Nouvelles approches du livre de Qohéleth. Avec une bibliographie (1988-1998) élaborée par Béatrice Perragaux Allisson, Fribourg/Göttingen, Vandenhœck \& Ruprecht (Orbis Biblicus et Orientalis, 168).

RotTzoll, D. V. (1999), Abraham Ibn Esras. Kommentare zu den Büchern Kohelet, Ester und Rut. Eingeleitet, übersetz und kommentiert, Berlin/New York, Walter de Gruyter. 
SACCHI, P. (20057), Qohelet (Ecclesiaste), Milan, San Paolo (Nuovissima versione della Bibbia, 20).

Saracino, F. (1982), "Ras Ibn Hani 78/20 and Some Old Testament Connexions ", Vetus Testamentum, 32, p. 339-340.

SAYler, G. D. (2001), Vain Rhetoric. Private Insight and Public Debate in Ecclesiastes, Sheffield, Sheffield Academic Press (Journal for the Study of the Old Testament. Supplement, 327).

Schoors, A. (2004), The Preacher Sought to Find Pleasing Words. A Study of the Language of Qoheleth. Part II. Vocabulary, Leuven, Peeters (Orientalia Lovaniensia Analecta, 143).

SCHWIENHORST-SCHÖNBERGER, L. (2004), Kohelet, Fribourg, Verlag (Herders Theologischer Kommentar zum Alten Testament).

SEOW, C.-L. (1997), Ecclesiastes. A New Translation with Introduction and Commentary, New York, Doubleday (Anchor Bible, 18c).

SHIELDS, M. A. (2006), The End of Wisdom. A Reappraisal of the Historical and Canonical Function of Ecclesiastes, Winona Lake, Eisenbrauns.

STrobel, A. (1967), Das Buch Prediger, Düsseldorf, Patmos-Verlag (Die Welt der Bibel. Kleinkommentare zur Heiligen Schrift, 9).

Taradach, M. et J. Ferrer (1998), Un Targum de Qohéleth. Éditio Princeps du LMS. M-2 de Salamanca. Texte araméen, traduction et commentaire critique, Genève, Labor et Fides (Le monde de la Bible, 37).

Traduction oecuménique de la Bible (1975), Paris, Cerf/Les Bergers et les Mages.

Vilchez Lindez, J. (1994), Sapienciales III. Ecclesiastes o Qohelet, Navarre, Editorial Verbo Divino.

Whybray, R. N. (1989), Ecclesiastes, Grand Rapids, Eerdmans.

WiLlmes, B. (2000), Menschliches Schicksal und Ironische Weisheitkritik im Koheletbuch. Kohelets Ironie und die Grenzen der Exegese, Neukirchener, Verlag (Biblisch-Theologische Studien, 39).

Zimmerli, W. (1980), Das Buch des Predigers Salomo, Göttingen, Vandenhœck \& Ruprecht (Das Alte Testament Deutsch, 16/1,2). 


\section{Résumé}

Qo 10,1 est un texte qui ne nécessite aucune correction textuelle. Du point de vue de l'analyse structurelle, Qo 9,18b-10,1a est un commentaire critique de Qo 9,18a, tandis que Qo 10,1b s'oppose à Qo 9,17. L'idée dominante de ce verset peut se résumer comme suit: Qo 10,1 relativise la valeur traditionnelle de la sagesse. Plus précisément, Qo 9,18b-10,1a évoque le monde politico-militaire qui a le pouvoir de détruire le bonheur, tandis que Qo 10,1b souligne qu'un peu de folie a plus de valeur que la sagesse et la gloire. Le mot clé est ici $m^{\prime} t$, « un peu ». Il rappelle, non sans ironie, que ni la sagesse ni la folie ne peuvent garantir le bonheur et la sécurité (cf. Qo 7,16-17).

\section{Abstract}

Qo 10,1 is a text that doesn't require textual correction. From the standpoint of structural analysis, Qo 9,18b-10,1a is a critical commentary of Qo 9,18a, whereas Qo 10,1b is opposed to Qo 9,17. The main thought behind this verse can be summarized as follows: Qo 10,1 contextualizes the traditional value of wisdom. More precisely, Qo 9,18b-10,1a evokes the power of the political and military world to destroy happiness, whereas Qo 10,1b underlines that a little folly is more worthy than wisdom and glory. Here, the key word is $m^{\prime} t$, " a little». It reminds us, not without irony, that neither wisdom nor folly can guarantee happiness and security (cf. Qo 7,16-17). 